Check for updates

Cite this: RSC Adv., 2017, 7, 37675

Received 7th June 2017

Accepted 19th July 2017

DOI: $10.1039 / c 7 r a 06386 e$

rsc.li/rsc-advances

\section{Slide cover glass immobilized liquid crystal microdroplets for sensitive detection of an IgG antigen $\uparrow$}

\author{
Yue Huan, ${ }^{a}$ So Jung Park, ${ }^{a}$ Kailash Chandra Gupta, ${ }^{\text {ab }}$ Soo-Young Park (D) a \\ and Inn-Kyu Kang (D) *a
}

Rabbit IgG antigen sensing liquid crystal (LC) microdroplets with a size variation from 15-20 $\mu \mathrm{m}$ were prepared using $98 \%$ nematic 4-cyano-4'-pentyl biphenyl (5CB), poly(styrene-b-acrylic acid) copolymer (PS-b-PA), and sodium dodecyl sulfate (SDS) as an interfacial modifier. The slide cover glass immobilized AlgG conjugated LC microdroplets were found to be sensitive in showing optical signals on interactions with an IgG antigen both in phosphate-buffered saline solution (PBS) and in the presence of other media i.e., $10 \mathrm{wt} \%$ fetal bovine serum (FBS) and blood plasma. The slide cover glass has enhanced the possibilities of efficient interactions of the IgG antigen with immobilized AlgG conjugated LC microdroplets and decreased the lower limit of detection for the $\mathrm{lgG}$ antigen down to $25 \mathrm{ng} \mathrm{mL}^{-1}$ within a contact time of $30 \mathrm{~min}$ in PBS solution at room temperature. The sensitivity of cover-glassimmobilized LC microdroplets in detection of IgG in solution with 10 wt\% amount of FBS and blood plasma is found to be same ( $25 \mathrm{ng} \mathrm{mL}^{-1}$ of $\mathrm{lgG}$ ) as found in PBS solution on comparing the response of slide cover glass immobilized LC microdroplets for $\mathrm{lg} G$ at $~ 50 \%$ orientation transitions in LC microdroplets. However, the response time of slide-cover-glass-immobilized LC microdroplets for detection of IgG in solution with 10 wt\% FBS or blood plasma is delayed to 60 minutes. The slide-coverglass-immobilized LC microdroplets have also shown excellent storage stability and reuse applicability in sensitive detection of IgG in biological simulated fluids.

\section{Introduction}

Proteins are essential components of vascular and tissue systems, and able to control a wide range of biological activities in living systems. The abnormal expression of a protein is often associated with a specific type of disease in living organisms; ${ }^{1}$ hence, sensitive and rapid detection of protein markers or antigens is quite useful for diagnosis and treatment of diseases at an early stage. ${ }^{2-4}$ However, the diseaserelated protein biomarkers or antigens are always at ultralow levels in early stages of chronic diseases, which makes their detection very difficult with conventional methods. ${ }^{5}$ In this direction, enormous efforts have already been made to develop techniques for sensitive detection of proteins such as, surface-enhanced Raman scattering, ${ }^{6}$ microfluidics, ${ }^{7}$ and

${ }^{a}$ Department of Polymer Science and Engineering, Kyungpook National University, 80, Daehak-ro, Buk-gu, Daegu 702-701, South Korea.E-mail: ikkang@knu.ac.kr; psy@ knu.ac.kr; 20100819@hanmail.net; sojung90714@naver.com; Fax: +82-53-9506623; Tel: +82-53-950-5629

${ }^{b}$ Polymer Research Laboratory, Department of Chemistry, I.I.T. Roorkee, Roorkee 247667, India. E-mail: kcgptfcy@iitr.ac.in; Fax: +91 1332 273560; Tel: +91 1332 285325

$\dagger$ Electronic supplementary information (ESI) available. See DOI: 10.1039/c7ra06386e electrochemiluminescence assaying. ${ }^{8}$ In detection of disease causing proteins, the conventional mass spectrometry is redundant in providing the required information particularly for the presence of ultralow amounts of proteins in biological fluids. Nevertheless, it has recently been applied for a sensitive detection of metabolite but detection of ultra low amount of proteins is still found to be difficult using mass spectrometry. ${ }^{9,10}$ For ultrasensitive detection of low-amount of protein biomarkers, the technique of signal amplification through biomarker tagging on a solid surface is proved to be somewhat useful. $^{11,12}$ The mass tagging method using immobilized antibody or aptamer ${ }^{13}$ is found easier in detection of antigens in flowing liquid in comparison to detecting systems having both antibody and antigen in single mobile phase. This might be a basis for immobilization of AIgG antibody conjugated LC microdroplets on cover glass for sensitive detection of antigen in liquid media. The solid surface immobilized antibodies on indium tin oxide (ITO) were found to be efficient in providing stable interactions to circulating antigens or pathogens due to the presence of directional orientations of immobilized antibodies. The surface immobilized antibodies also found useful in reducing non-specific interactions with other biomolecules or antigens; hence, they are found to be more efficient in assaying biomolecules accurately without any interference 
from other molecules in the media. In addition to sensitivity enhancement for detection of antigens by solid surface immobilized antibodies, they have also shown a significant reduction in sensing time for the detection of antigens due to rapid and effective interactions with antibodies without fluorescent labeling of cells or antigens. In solid surface immobilized antibody technique, the unwanted and physically adsorbed impurities are washed out easily; hence, the response of immobilized antibody for detection of antigens or other molecules has shown a significant improvement. The ITO solid surface immobilized anti-CD33 antibody is found quite effective in detection of CD33 antigen on human promyelocytic leukemia cells within a response time of $30 \mathrm{s.}^{\mathbf{1 4}}$ The method of surface immobilization of antibodies is also found useful in simultaneous detection of various antigens by using an array of antibodies on solid surfaces. ${ }^{15-18}$

In our previous studies, we were able to utilize the optical properties of liquid crystal (LC) microdroplets for in vitro detection of antigens ${ }^{\mathbf{1 9}}$ and $\mathrm{KB}$ cancer cells expressed with folate biomarkers ${ }^{20}$ by making use of antibody-antigen interactions induced optical transitions in 5CB molecules in LC microdroplets. $^{21-25}$ The selective affinity of antibodies for diseasecausing antigens has been used successfully in immunoassaying of SK-BR3 cancer cells ${ }^{26-31}$ and pathogenic proteins of bacteria and virus. ${ }^{32-34}$ In comparison to nanomaterial-based method of sensing, the solid surface immobilized LC microdroplet-method is found easier ${ }^{35-38}$ in detection of proteins as it does not require fluorescent labeling of target molecules and is able to produce optical signals by the interactions of antibody-protein at the interface of LC microdroplets. Thus considering the various advantages of solid surface immobilized antibodies in detection of proteins, an attempt has been made to immobilize the antibody conjugated LC microdroplets on slide cover glass for a sensitive detection of rabbit antigen (IgG) in liquid media using antibody-antigen interaction forces to cause optical transitions in 5CB molecules. To carry out these investigations, the anti-IgG conjugated LC microdroplets were immobilized on 3-aminopropyltriethoxysilane (APTES) treated slide cover glass and resultant antibody-antigen interactions induced optical transition in LC microdroplets has been used successfully for a sensitive detection of rabbit IgG antigen in liquid media and biological simulated fluids.

\section{Experimental}

\section{Materials and methods}

Circular cover glass (Borosilicate glass type D 263® M) with a thickness of $0.15 \mathrm{~mm}$ and $10 \mathrm{~mm}$ diameter were procured from Paul Marienfeld GmbH \& Co. KG, Lauda-Königshofen, Germany.

(3-Aminopropyl)triethoxysilane (APTES) as silane coupling agent $\left(M_{\mathrm{w}}, 221.37 \mathrm{~g} \mathrm{~mol}^{-1}\right)$, 4-cyano-4'-pentyl biphenyl liquid crystal with $98 \%$ nematic phase (5CB) $\left(M_{\mathrm{w}}, 249.15 \mathrm{~g} \mathrm{~mol}^{-1}, \mathrm{mp}\right.$ $24{ }^{\circ} \mathrm{C}$ ), sodium dodecylsulfate (SDS) $\left(M_{\mathrm{w}}, 288.38 \mathrm{~g} \mathrm{~mol}^{-1}\right)$, poly(styrene- $b$-acrylic acid) (PS- $b$-PA) $\left(M_{\mathrm{w}}, 7246 \mathrm{~g} \mathrm{~mol}^{-1}\right)$, di-tertbutylpyrocarbonate, 1-ethyl-3-(3-dimethyl aminopropyl) carbodiimide hydrochloride (EDC) $\left(M_{\mathrm{w}}, 312.38 \mathrm{~g} \mathrm{~mol}^{-1}\right), N$ hydroxysuccinimide (NHS) $\left(M_{\mathrm{w}}, 115.09 \mathrm{~g} \mathrm{~mol}^{-1}\right)$, and fluorescein isothiocyanate (FITC) were purchased from Sigma Aldrich Chemical Company, USA, and used as received. The phosphate buffered saline (PBS) solution of $\mathrm{pH} 7.4$ was used for washing and dispersion of LC microdroplets. The reagent grade, $>95 \%$ (SDS-PAGE), salt-free lyophilized white powdered rabbit antigen (IgG) $(150 \mathrm{kDa})$ was purchased from Sigma Aldrich Chemical Company, USA, and stored below $8{ }^{\circ} \mathrm{C}$ before using as a reference antigen for immunoassaying the pathogens (viruses and bacteria) by LC microdroplets. The unconjugated rabbit antiIgG (AIgG) ( $M_{\mathrm{w}}, 150 \mathrm{kDa}$, lyophilized white powder) was also purchased from Sigma Aldrich Chemical Company, USA. The AIgG, which was used in these experiments was specific to rabbit IgG and did not react with human immunoglobulin-G (IgG). The FITC-AIgG conjugate was synthesized to anchor on slide cover glass immobilized LC microdroplets and all measurements were carried out in PBS solution. The ultrapure water was used in experimental work and was prepared using Milli-Q system. The UV-vis spectra were recorded using double beam UV-vis spectrophotometer (Jasco-650, USA). The size of LC microdroplets and their size distribution was determined using optical microscope (Nikon Eclipse TS100, Japan) and a particle size analyzer (Beckman coulter, N/LS-1332, USA). The optical and polarized light micrographs of LC microdroplets were recorded using Olympus IX 71 inverted fluorescence microscope using both cross-polarization and transmission modes. An upright fluorescence microscope (Olympus BX61, Olympus America Inc., USA) with a fluorescent filter tube above the objective lenses, coupled with a digital camera, was used for the detection of FITC and its conjugate $\left(\lambda_{\mathrm{EX}}=519 \mathrm{~nm}\right)$. The X-ray photoelectron spectrometer (ESCA, ESCA LAB VIG Microtech, East Grinstead, UK, using $\mathrm{Mg} \mathrm{K} \alpha$ radiation) was used to record the X-ray photoelectron spectra of APTES modified surface of slide cover glass to confirm the amino-functionalization.

\section{Preparation of LC microdroplets}

To prepare the LC microdroplets, $20 \mathrm{mg}$ (70 $\mu \mathrm{mol})$ of SDS was added to a $100 \mathrm{~mL}$ round-bottom flask containing $60 \mathrm{mg}$ (8.4 $\mu \mathrm{mol}$ ) block copolymers (PS- $b$-PA) dissolved in $20 \mathrm{~mL}$ PBS solution. The mixture was stirred magnetically for about $1 \mathrm{~h}$ at $500 \mathrm{rpm}$ to mix SDS thoroughly and obtain a homogeneous mixture of SDS with amphiphilic block copolymer (PS- $b$-PA). To prepare the LC microdroplets emulsion, $200 \mathrm{mg}(800 \mu \mathrm{mol})$ of 5CB LC (98\% nematic phase) was added drop-wise to a homogeneous solution of PS- $b$-PA and SDS. The resulting mixture was stirred continuously at $11000 \mathrm{rpm}$ for about $20 \mathrm{~s}$ to produce a homogeneous emulsion of LC microdroplets. The resultant emulsion was centrifuged at $800 \mathrm{rpm}$ in PBS solution ( $\mathrm{pH} 7.4$ ) to separate the LC microdroplets from the unused 5CB, SDS, and PS- $b$-PA. After centrifugation, the supernatant was discarded to remove the small-sized microdroplets. The centrifuged LC microdroplets were then dispersed in $20 \mathrm{~mL}$ PBS solution and used subsequently for anchoring on slide cover glasses after conjugating with AIgG in PBS solution. Finally, the slide-coverglass-immobilized LC microdroplets were used to study their 
interactions with IgG antigen in PBS solution and other media such as $10 \mathrm{wt} \%$ solutions of fetal bovine serum (FBS, Gibo, USA) and blood plasma (Sigma Aldrich Chemical Company). The size distribution of pristine LC microdroplets was determined using optical microscope and particle size analyzer. The orientational state of 5CB molecules in LC microdroplets was determined by recording optical and polarized light micrographs using Olympus IX 71 inverted fluorescence microscope operating in both cross-polarization and transmission modes. The optical texture of LC microdroplets was recorded using the same fluorescence microscope. The presence of two-point defects (boojums) in optical texture of LC microdroplets indicated a bipolar state of 5CB molecules in LC microdroplets and one-point defect (boojum) is taken to indicate a radial state of $5 \mathrm{CB}$ molecules in LC microdroplets. The optical texture variation method is applied for detection of orientation change in liquid crystal molecules confined in droplets because it is difficult to differentiate the variation in azimuthal orientation of liquid crystal molecules in microdroplets in comparison to determination of variation in azimuthal orientation of liquid crystal molecules on planar solid surfaces. Therefore, optical texture method has been used for detection of change in orientation of LC molecules in microdroplets.

\section{Surface modification of slide cover glasses by APTES}

The acetone-cleaned microscopic slide cover glasses were treated with piranha solution $\left(1: 3,30 \% \mathrm{H}_{2} \mathrm{O}_{2}: 3 \mathrm{H}_{2} \mathrm{SO}_{4}\right)$ for approximately $30 \mathrm{~min}$ before use. After removing the excess unused piranha solution by washing with distilled water, the slide cover glasses were allowed to react with $2 \mathrm{wt} \%$ aqueous solution of APTES in 95\% acetone for $3 \mathrm{~min}$ at $11.2 \mathrm{pH}$. Finally, the slide cover glasses were rinsed 3 times with ethanol and dried under vacuum. To confirm the amino functionalization of the surface of slide cover glasses by APTES, the contact angle measurements and X-ray photoelectron spectrometric analysis of slide cover glass was carried out by using sessile drop method of contact angle measurements using a ráme-hart goniometer (Mountain Lakes, NJ, USA) and XPS spectra were recorded using ESCALAB MK II X-ray photoelectron spectrometer (VG Scientific Ltd., England), respectively.

\section{Anchoring of LC microdroplets on amino functionalized slide cover glasses}

To enhance the sensitivity and interactions of LC microdroplets with IgG antigen, the purified LC microdroplets having size variation from 15-20 $\mu \mathrm{m}$ were immobilized using APTES modified slide cover glasses. To immobilize the LC microdroplets on APTES modified slide cover glasses, the carboxylic groups of amphiphilic block copolymer of LC microdroplets were activated by drop-wise addition of $1.0 \mathrm{~mL}$ of LC microdroplets emulsion $\left(18 \times 10^{3}\right.$ microdroplets per $\left.\mathrm{mL}\right)$ in a round bottom flask containing $200 \mathrm{mg}(640 \mu \mathrm{mol})$ of NHS and $200 \mathrm{mg}$ $(1740 \mu \mathrm{mol})$ of EDC dissolved in $20 \mathrm{~mL}$ PBS at $25{ }^{\circ} \mathrm{C}$. In this solution, the slide cover glasses were immersed and kept for overnight at $25^{\circ} \mathrm{C}$. After $12 \mathrm{~h}$, the slide cover glasses were taken out from activating mixture and washed with distilled water to remove unused reagents and free LC microdroplets. After washing, the LC microdroplets immobilized slide cover glasses were preserved in PBS solution ( $\mathrm{pH}$ 7.4) to conjugate AIgG antibody and for recording the orientation variation in 5CB molecules on interactions with IgG antigen. To confirm the immobilization and positional stability of LC microdroplets on slide cover glasses, the optical microscopic images of stabilized and free LC microdroplets on slide cover glass were captured at a time interval of $1 \mathrm{~min}$ for a total time of $10 \mathrm{~min}$ and relative separation between any two droplets was determined.

\section{Conjugation of AIgG antibody on slide cover glass immobilized LC microdroplets}

To add antigen-specific selectivity to cover glass immobilized LC microdroplets, the AIgG antibody was conjugated with slide cover glass immobilized LC microdroplets. To conjugate AIgG on slide cover glass immobilized LC microdroplets, first of all, the slide-cover-glass-immobilized LC microdroplets were immersed in a $100 \mathrm{~mL}$ round bottom flask containing $200 \mathrm{mg}$ $(640 \mu \mathrm{mol})$ of NHS and $200 \mathrm{mg}(1740 \mu \mathrm{mol})$ of EDC dissolved in $20 \mathrm{~mL}$ PBS at $25{ }^{\circ} \mathrm{C}$. To this solution, $5 \mathrm{~mL}$ of PBS solution containing $50 \mu \mathrm{g}(34 \mathrm{pmol})$ of AIgG was added slowly and resultant mixture was kept overnight at $25{ }^{\circ} \mathrm{C}$. After $12 \mathrm{~h}$, the slide cover glass having AIgG-conjugated LC microdroplets were taken out from activating mixture and washed with distilled water to remove the unused AIgG and other reagents. The amount of AIgG conjugated with LC microdroplets was determined by Bradford method using a calibration curve, which was drawn at $\lambda_{\max }$ of $280 \mathrm{~nm}$ taking different amounts of AIgG in PBS solution.

\section{Conjugation of AIgG-FITC on slide cover glass immobilized LC microdroplets}

To confirm the conjugation of AIgG on slide cover glass immobilized LC microdroplets, the synthesized AIgG-FITC conjugate was immobilized on LC microdroplets and fluorescence images of AIgG-FITC conjugated LC microdroplets were recorded. To conjugate the AIgG-FITC on slide cover glass immobilized LC microdroplets, first of all AIgG-FITC was synthesized by drop-wise addition of $10 \mathrm{~mL}$ of PBS solution containing $50 \mu \mathrm{g}$ ( $34 \mathrm{pmol})$ of AIgG to a $100 \mathrm{~mL}$ round bottom flask containing $200 \mathrm{mg}$ each of EDC and NHS dissolved in 10 $\mathrm{mL}$ of PBS solution to activate the carboxyl groups of AIgG. To this solution, a freshly prepared $10 \mathrm{~mL}$ solution of FITC $(50 \mu \mathrm{g})$ in DMSO (dimethyl sulfoxide) was added and the mixture was left in dark at $5{ }^{\circ} \mathrm{C}$. After $8 \mathrm{~h}$, the reaction mixture was dialyzed with regenerated cellulose acetate membranes (MWCO, $12 \mathrm{kDa}$ ) using PBS solution. Finally, AIgG-FITC conjugate was washed with PBS solution and obtained as a white powdery mass after lyophilization. The conjugation of FITC with AIgG, was confirmed by recording UV-vis spectra of FITC, AIgG, and FITCAIgG conjugate using UV-vis spectrophotometer. To anchor AIgG-FITC conjugate on cover glass immobilized LC microdroplets, a $10 \mathrm{~mL}$ PBS solution containing $50 \mu \mathrm{g}$ of AIgG-FITC conjugate was added to a $100 \mathrm{~mL}$ round bottom flask containing slide-cover-glass-immobilized LC microdroplets and activating 
agents (EDC and NHS, $100 \mathrm{mg}$ each). The mixture of AIgG-FITC conjugate and immobilized microdroplets was incubated for $4 \mathrm{~h}$ at $5{ }^{\circ} \mathrm{C}$. Finally, the solution was dialyzed with regenerated cellulose acetate membrane (MWCO, $12 \mathrm{kDa})$ using PBS (pH, 7.4) solution. The cover glass immobilized LC microdroplets conjugated with AIgG-FITC were washed with PBS solution to remove unused AIgG-FITC and other activating reagents. The anchoring of AIgG-FITC conjugate on cover glass immobilized LC microdroplets was confirmed by recording fluorescent images of immobilized LC microdroplets $\left(\lambda_{\mathrm{EX}}=519 \mathrm{~nm}\right) \mathrm{using}$ upright fluorescence microscope (Olympus BX61, Olympus America Inc., USA) with fluorescent filter tube above the objective lenses, coupled with a digital camera. The orientational state of 5CB molecules in AIgG-FITC-immobilized LC microdroplets was examined by recording polarized light micrographs using Olympus IX 71 inverted fluorescence microscope, operating in both cross-polarization and transmission modes.

\section{Detection of IgG antigen using slide cover glass immobilized AIgG-conjugated LC microdroplets}

The response of slide cover glass anchored AIgG-conjugated LC microdroplets for IgG antigen in PBS ( $\mathrm{pH}$ 7.4) solution was evaluated by immersing gently one piece of AIgG-conjugated LC microdroplets immobilized slide cover glass in a circular dish of $2.0 \mathrm{~cm}$ diameter containing $1.0 \mathrm{~mL}$ solution of IgG $(50$ $\mathrm{ng} \mathrm{mL} \mathrm{m}^{-1}$ ) at room temperature without touching the surface of slide cover glass. To optimize the contact time of slide cover glass immobilized AIgG-conjugated LC microdroplets, the slide cover glass immobilized AIgG-conjugated LC microdroplets were allowed to interact with IgG antigen for different time intervals and variation in orientational state of $5 \mathrm{CB}$ molecules in LC microdroplets was recorded using polarized light micrographs. To compare the effect of immobilization of LC microdroplets on slide cover glass, the experiments were also carried out using free AIgG-conjugated LC microdroplets $(18 \times$ $10^{3}$ microdroplets per $\mathrm{mL}$ ) in presence of same concentration

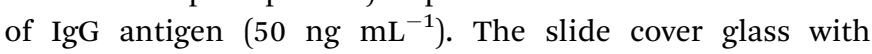
homogeneously distributed LC microdroplets was used to optimize the contact time of LC microdroplets for detection of IgG antigen. After optimizing the contact time of immobilized LC microdroplets, the experiments were also repeated by keeping slide cover glass immobilized LC microdroplets for optimized contact time in solutions having different concentrations of IgG ranging from $10-50 \mathrm{ng} \mathrm{mL}^{-1}$ at room temperature and orientational state of $5 \mathrm{CB}$ molecules in LC microdroplets was determined. In order to simulate the response of immobilized LC microdroplets for a real system, the optical response of slide cover immobilized LC microdroplets for IgG antigen was also determined in presence of 10 wt $\%$ solution of FBS blood plasma. The optical response of immobilized LC microdroplets for IgG antigen both in $10 \mathrm{wt} \%$ solution of FBS and blood plasma was compared with the response of LC microdroplets in PBS solution. Finally, the results were analyzed critically to explain the variation in optical response of AIgG anchored LC microdroplets.

\section{Results and discussion}

The immobilization of AIgG-conjugated LC microdroplets on slide cover glass is found an useful method to develop immunobiosensor with sustained dispersity of LC microdroplets and constant response time for in vitro detection of IgG antigen in liquid media. The cover glass immobilized LC microdroplets have shown consistent optical signal by maintaining a stable contact with IgG antigen until IgG-AIgG interactions were transduced fully to an optically detectable signal. To achieve enhanced interactions between AIgG conjugated LC microdroplets and IgG antigen, the density of LC microdroplets on cover glass surfaces is found to be of great significance and has been controlled by taking sufficient amount of APTES during amino functionalization of slide cover glass. The increased density $(2988 \pm 2.0$ LC microdroplets per cover glass) and proper orientation of LC microdroplets on slide cover glass are considered responsible for the increased sensitivity of LC microdroplets in detection of IgG in comparison to free and randomly oriented LC microdroplets used in test solution. Therefore, slide cover glass were amino functionalized very carefully by taking optimized amount of APTES (5 wt\%) to control the density and orientation of LC microdroplets and their subsequent conjugation with AIgG antibody to interact selectively with IgG antigen. The proper orientation of AIgG conjugated LC microdroplets on slide cover glass has contributed significantly for the enhanced antibody-antigen interactions and to induce optical transitions in LC microdroplets even in presence of very low amount of IgG antigen $\left(>10 \mathrm{ng} \mathrm{mL}^{-1}\right)$ in liquid media. The optical transitions in LC microdroplets were only possible on proper interactions of IgG antigen with LC microdroplets conjugated AIgG and on producing sufficient force of interactions at the interface of LC microdroplets. Improper and weak interactions of IgG antigen with LC microdroplets conjugated AIgG remained ineffective in showing optical transitions in LC microdroplets. The slide cover glass immobilized LC microdroplets were found to be more sensitive as cover glass has provided ample opportunities to IgG antigen to interact effectively with LC microdroplets anchored AIgG even at low concentration and in presence of other interfering protein molecules, which were present in $10 \mathrm{wt} \%$ solution of blood plasma and FBS.

\section{Preparation and characterization of pristine LC microdroplets}

In order to add sensitivity to 5CB molecules in detection of interfacial interaction forces, the $5 \mathrm{CB}$ molecules in the form of microdroplets are found useful with enhanced interfacial interactions to antigens. ${ }^{36,39-44}$ Various studies have indicated that 5CB molecules in the form of LC microdroplets were found useful in developing naked-eye-detectable optical signals on interactions with virus and bacteria. The 5CB molecules in the form of microdroplets were found to be efficient in providing sufficient interfacial area for interactions as similar to nano materials based sensors. ${ }^{45,46}$ The LC microdroplets anchored with small amount of AIgG $\left(50 \mu \mathrm{g} \mathrm{mL}^{-1}\right)$ were found capable to show detectable signals for interactions with antigen. Moreover, the 5CB molecules in the form of LC microdroplets have shown a capacity for detection of IgG concentration in solution as low 


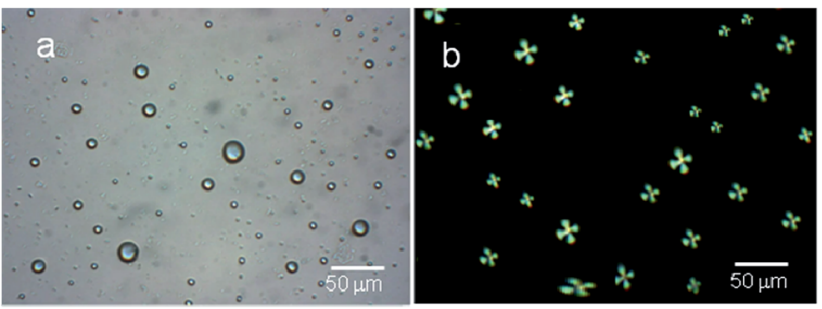

Fig. 1 Optical (a), and polarized (b) light micrographs of LC microdroplets.

as $>10 \mathrm{ng} \mathrm{mL}^{-1}$ in comparison to layered structures of $5 \mathrm{CB}$ molecules on solid planar surfaces. The layered structures of 5CB molecules on solid planar surfaces were able to create detectable signals in presence of microgram amount of analytes. ${ }^{38,47,48}$ These studies have suggested that 5CB molecules in the form of LC microdroplets are more sensitive and able to show detectable signal easily in comparison to layered structures on solid planar surfaces.

To study the application of LC microdroplets in detection of IgG, an emulsion of LC microdroplets was prepared by adding $200 \mathrm{mg}(400 \mu \mathrm{mol})$ of $5 \mathrm{CB}$ in a homogenized (11 $000 \mathrm{rpm})$ aqueous dispersion containing $20 \mathrm{mg}(70 \mu \mathrm{mol}) \mathrm{SDS}$ and $60 \mathrm{mg}$ $(2.8 \mu \mathrm{mol})$ PS- $b$-PA in $20 \mathrm{~mL}$ PBS solution. The prepared LC microdroplets have shown a size variation from $15-20 \mu \mathrm{m}$ as confirmed from the optical images recorded using $1 \mathrm{~mL} \mathrm{LC}$ microdroplets dispersions in a dish of $1 \mathrm{~cm}$ diameter (Fig. 1a).

The prepared LC microdroplets were characterized for the orientations of $5 \mathrm{CB}$ molecules, which found to be with radial configuration as confirmed from polarized light micrographs (Fig. 1b).

\section{Surface modification of slide cover glass by 3-aminopropyltriethoxysilane}

The circular slide cover glasses of $1.0 \mathrm{~cm}$ diameter were used for the immobilization of LC microdroplets after amino functionalization with 3-aminopropyltriethoxysilane (APTES). Before amino functionalization, the slide cover glasses were cleaned with acetone and then treated with piranha solution $\left(1: 3, \mathrm{H}_{2} \mathrm{O}_{2}\right.$ $(30 \%): 3 \mathrm{H}_{2} \mathrm{SO}_{4}$ ) for approximately $30 \mathrm{~min}$, which produced cover glass with sufficient amount of hydroxyl groups (Scheme 1a). The surface of slide cover glass was treated with 5 wt\% solution of APTES in acetone so that maximum hydroxyl groups were reacted to APTES to create slide cover surface with high density of amino groups (Scheme 1b). The amino functionalization of slide cover glass was confirmed by comparing the contact angles of piranha treated cover glass with amino functionalized cover glass after treating with $2 \mathrm{wt} \%$ solution of APTES.

The values of contact angles $(\theta)$ of slide cover glass have indicated that piranha treated cover glass were having more wettability as the values of contact angle $(\theta)$ was decreased to $34.61^{\circ}$ in comparison to original contact angle of $53.25^{\circ}$ (Fig. 2a and $b$ ).

However, on reacting hydroxyl-group-functionalized slide cover glass with $2 \mathrm{wt} \%$ solution of APTES, the value of contact angle of APTES-treated slide cover glass is increased to $85.36^{\circ}$ (Fig. 2c), which contradicted the expected formation of hydrophilic surface due to the formation of terminal hydrophilic amino groups on treating with $2 \mathrm{wt} \%$ solution of APTES. These results have indicated that the amount of APTES used for the amino functionalization was slightly less than what was required for total functionalization of hydroxyl groups or reaction time of 3 min was insufficient for the reaction of APTES with hydroxyl groups at $\mathrm{pH}$ 11.2. This caused the formation of hydrophobic surfaces of slide cover glass due to hydrogen bonding between residual hydroxyl groups on cover glass with newly form amino groups by APTES. Thus hydrogen bonding might be a reason for the formation of hydrophobic surface of slide cover glass on using $2 \mathrm{wt} \%$ solution of APTES, which is in agreement with the studies reported by other workers. ${ }^{49,50}$ To confirm the amino functionalization of slide cover glass by APTES, the X-ray photoelectron spectra of amino functionalized cover glass were recorded using ESCALAB MK II X-ray
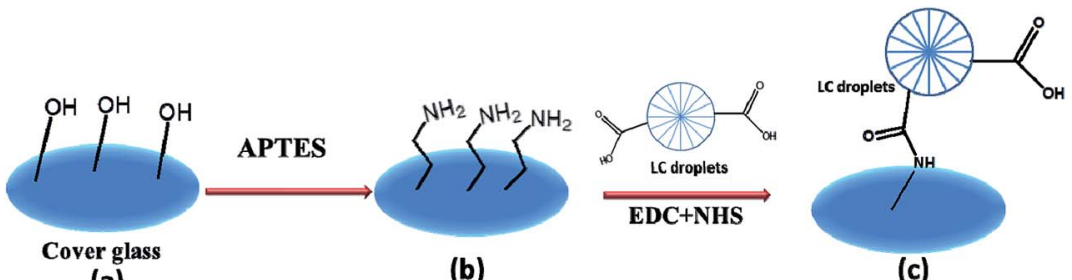

(a)

(b)

(c)

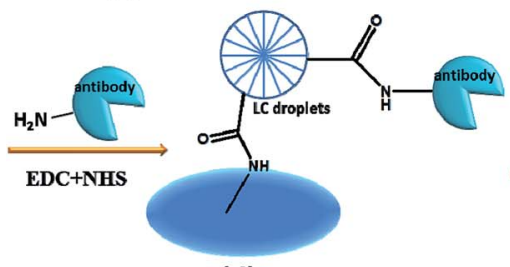

(d)

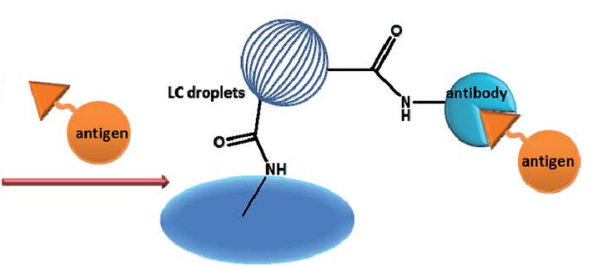

(e)

Scheme 1 Cover glass (a) functionalization and immobilization of AlgG conjugated LC microdroplets on aminofunctionalized cover glass (b). Orientation state of 5CB molecules in LC microdroplets on anchoring at slide cover glass (c) and on interactions with AlgG (d), and with IgG antigen in PBS solution (e). 


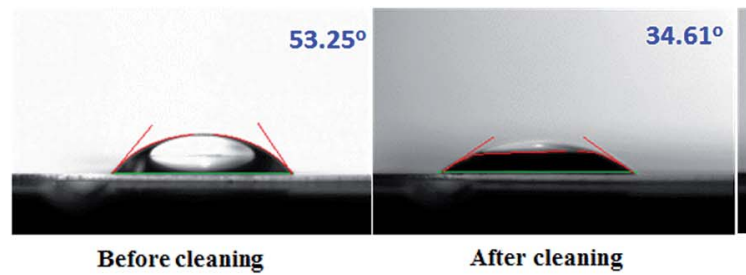

(a)

(b)

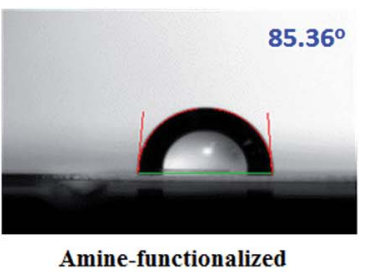

(c)

Fig. 2 Contact angle measurements of slide cover glass (a) before, (b) after treating with piranha solution, and (c) after reacting with 2 wt\% solution of APTES.

photoelectron spectrometer (VG Scientific Ltd., England). The $\mathrm{X}$-ray photoelectron spectra and elements survey data for piranha and APTES-treated slide cover glass are shown in Fig. 3 and Table 1 . The characteristic signals for $2 \mathrm{p}$ and $2 \mathrm{~s}$ for glass silica appeared at $104 \mathrm{eV}$ and $154 \mathrm{eV}$, respectively, whereas; a prominent peak at $532 \mathrm{eV}$ was for binding energy of $\mathrm{O} 1 \mathrm{~s}$. The cleaned cover glass (Fig. 3a) did not show any signal for binding energy for nitrogen but APTES treated cover glass (Fig. 3b-d) have shown a peak for binding energy for $\mathrm{N} 1 \mathrm{~s}$ at $400 \mathrm{eV}$, which clearly indicated for amino functionalization of cover glass by the reaction of APTES.

However, no significant variation in intensity of $\mathrm{N} 1 \mathrm{~s}$ peak noticed on varying the amount of APTES from 5-10 wt\% (Fig. 3 and Table 1). Thus X-ray photoelectron spectra of piranhasolution-treated slide cover glass recorded at different amount of APTES have confirmed that $2.0 \mathrm{wt} \%\left(20 \mathrm{mg} \mathrm{mL}^{-1}\right)$ solution of APTES was insufficient for amino functionalization of piranhasolution-treated slide cover glass to fully transform the available hydroxyl groups into amino groups, therefore, residual hydroxyl groups were involved in formation of hydrogen bonding with amino group on cover glass and caused hydrophobicity ${ }^{49,50}$ as indicated by contact angle $\left(85.36^{\circ}\right)$ measurements. On further increasing the amount of APTES beyond $5.0 \mathrm{wt} \%$, the nitrogen content found to be same $(4.75 \%)$ as it was at $5.0 \mathrm{wt} \%$ APTES, which clearly indicated that all the hydroxyl groups on cover glass surfaces were saturated at $5.0 \mathrm{wt} \%$ solution of APTES, and there was no further consumption of APTES.

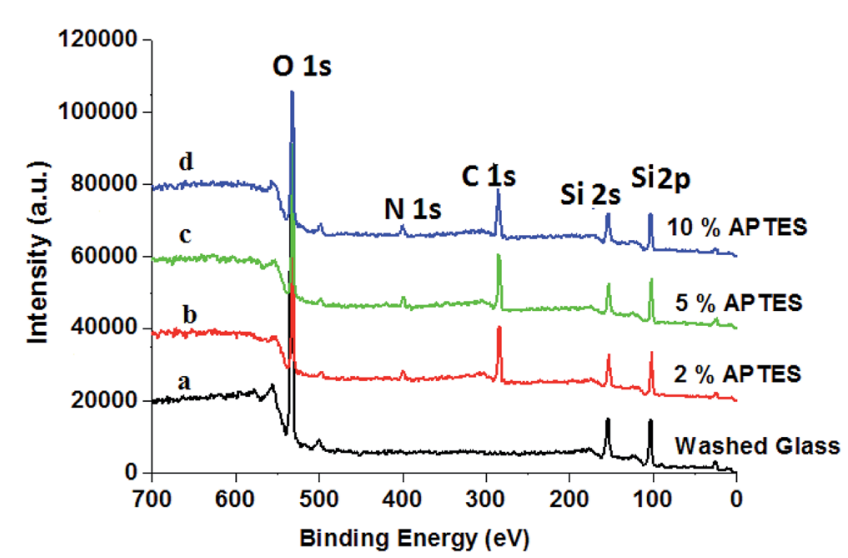

Fig. 3 X-ray photoelectron spectra of (a) cleaned cover glass, and ( $b-$ d) cover glass treated with different amount of APTES.
The anchored amount of APTES on each cover glass is found to be $3.6 \pm 0.02 \mathrm{mmol}$, which clearly suggested that each cover glass was having sufficient amount of amino groups for anchoring of LC microdroplets on cover glass.

\section{Immobilization of AIgG-conjugated LC microdroplets on amino-functionalized slide cover glass and their characterization}

To prepare the slide cover glass immobilized with LC microdroplets for the sensing of IgG antigen, the AIgG antibodyconjugated LC microdroplets were first coupled with aminofunctionalized slide cover glass. To couple LC microdroplets with amino-functionalized cover glass, the acrylic acid (PA) of amphiphilic PS- $b$-PA block copolymer of LC microdroplets was activated by mixing $1.0 \mathrm{~mL}$ of LC microdroplets emulsion $(18 \times$ $10^{3}$ microdroplets per $\mathrm{mL}$ ) in $20 \mathrm{~mL}$ PBS solution containing NHS $(640 \mu \mathrm{mol})$ and EDC $(1740 \mu \mathrm{mol})$ at $25^{\circ} \mathrm{C}$. To this solution, amino-functionalized slide cover glasses were added at $25{ }^{\circ} \mathrm{C}$. After $12 \mathrm{~h}$, LC microdroplets immobilized cover glasses (Scheme 1c) were removed and washed with PBS solution to remove unused reagent. Subsequently, the density of immobilized LC microdroplets on amino-functionalized slide cover glasses and orientational state of 5CB molecules in the LC microdroplets were determined. The efficiency of immobilization of LC microdroplets on slide cover glasses is found to be $83 \%$ (Table 2), and the density of immobilization of LC microdroplets on cover glass is found to be $3735 \pm 2.0$ LC microdroplets per $\mathrm{cm}^{2}(2988 \pm 2.0 \mathrm{LC}$ microdroplets per glass cover $)$ as determined by hemacytometer (Scheme 1c).

To positional stability of immobilized and free LC microdroplets on cover glass surfaces was compared by capturing the optical microscopic images of LC microdroplets at different

Table 1 Surface composition of cover glass with and without APTES by XPS

\begin{tabular}{llll}
\hline & \multicolumn{3}{l}{ Atomic percentage of elements } \\
\cline { 2 - 4 } Samples & $\mathrm{C} 1 \mathrm{~s}$ & $\mathrm{O} \mathrm{1s}$ & $\mathrm{N} 1 \mathrm{~s}$ \\
\hline Washed glass & 14.37 & 85.63 & - \\
2.0\% APTES & 46.58 & 48.83 & 4.59 \\
$5.0 \%$ APTES & 43.52 & 51.73 & 4.75 \\
$10.0 \%$ APTES & 43.56 & 51.37 & 4.75
\end{tabular}


Table 2 Specification of slide cover glass immobilized LC microdroplets in detection of IgG antigen in PBS solution (pH 7.4) and in solution with $10 \mathrm{wt} \%$ FBS or blood plasma ${ }^{a}$

\begin{tabular}{|c|c|c|c|}
\hline Specifications & PBS solution & FBS solution & Blood plasma \\
\hline LC microdroplets size distribution & $15-20 \mu \mathrm{m}$ & $15-20 \mu \mathrm{m}$ & $15-20 \mu \mathrm{m}$ \\
\hline Working range & $10-50 \pm 2.0 \mathrm{ng} \mathrm{mL}^{-1}$ & $10-50 \pm 2.0 \mathrm{ng} \mathrm{mL}^{-1}$ & $10-50 \pm 2.0 \mathrm{ng} \mathrm{mL}^{-1}$ \\
\hline Conjugation efficiency of AIgG/cover glass & $88 \%(8.8 \mu \mathrm{g})$ & $88 \%(8.8 \mu \mathrm{g})$ & $88 \%(8.8 \mu \mathrm{g})$ \\
\hline Conjugation efficiency of AIgG/LC microdroplets & $88 \%(2.95 \mathrm{ng})$ & $88 \%(2.95 \mathrm{ng})$ & $88 \%(2.95 \mathrm{ng})$ \\
\hline Lower limit of detection (LOD) for IgG/cover glass & $25 \pm 0.12 \mathrm{ng} \mathrm{mL}^{-1}$ & $25 \pm 0.16 \mathrm{ng} \mathrm{mL}^{-1}$ & $25 \pm 0.18 \mathrm{ng} \mathrm{mL}^{-1}$ \\
\hline LC microdroplets storage & 30 days & 30 days & 30 days \\
\hline
\end{tabular}

time intervals (Fig. S1 $\dagger$ ). The optical microscopic images of free and immobilized LC microdroplets at zero time and after $10 \mathrm{~min}$ have indicated a significant increase in separation in free microdroplets from initial separation of $65 \mu \mathrm{m}$ (Fig. S1a $\dagger$ ) to approximately $80 \mu \mathrm{m}$ (Fig. S1 $\mathrm{b}_{\dagger}^{\dagger}$ ) within a time interval of $10 \mathrm{~min}$. On the other hand, the separation between two immobilized LC microdroplets remained constant $(78 \mu \mathrm{m})$ after a time interval of $10 \mathrm{~min}$ (Fig. S1c-e $\dagger$ ). This result has indicated that immobilized LC microdroplets were having positional stability on cover glass as compared to free LC microdroplets, which on storing for $24 \mathrm{~h}$ have shown agglomeration in LC microdroplets (Fig. S1f $\dagger$ ). As antibody-antigen interactions found to be more effective with immobilized LC microdroplets; hence immobilized LC microdroplets were found to show rapid orientation transition in 5CB molecules in LC microdroplets in comparison to free LC microdroplets. The strong and effective antibody-antigen interactions are considered responsible for the increased sensitivity of immobilized LC microdroplets in detection of IgG as compared to free LC microdroplets as found in our earlier research ${ }^{19}$ and by other workers. ${ }^{38,47,48}$

The slide cover glasses with immobilized LC microdroplets were examined by polarized light microscopic images to evaluate the orientational state of 5CB molecules (Fig. 4).

The results have indicated that anchoring of LC microdroplets on slide cover glass (Fig. 4b) did not induce any transition in orientational state of 5CB molecules but 5CB molecules remained in radial orientation like pristine free LC microdroplets (Fig. 4a, Scheme 1c and d). It is presumed that the anchoring force of interactions of LC microdroplets on amino-functionalized cover glasses was not sufficient to influence the orientation of 5CB molecules in LC microdroplets; hence, 5CB molecules in LC microdroplets remained in radial configuration (Fig. 4b \& Scheme 1). To add selectivity to slide cover glass immobilized LC microdroplets to IgG antigen, the cover glass immobilized LC microdroplets were conjugated with AIgG by keeping LC microdroplets immobilized slide cover glass in a flask containing $200 \mathrm{mg}(640 \mu \mathrm{mol})$ of NHS and $200 \mathrm{mg}(1740 \mu \mathrm{mol})$ of EDC dissolved in $20 \mathrm{~mL}$ PBS solution ( $\mathrm{pH}$ $=7.4)$. After adding $50 \mu \mathrm{g}(34 \mathrm{pmol})$ of AIgG in flask, the mixture was kept at $25{ }^{\circ} \mathrm{C}$ (Scheme 1e). After $12 \mathrm{~h}$, the mixture was dialyzed with PBS ( $\mathrm{pH}=7.4)$ solution using regenerated cellulose acetate membrane (MWCO, $12 \mathrm{kDa}$ ) and slide cover glass were washed with distilled water. The polarized light images of AIgG conjugated LC microdroplets immobilized on slide cover glass were recorded to examine the orientational state of $5 \mathrm{CB}$ molecules in LC microdroplets before and after conjugation with AIgG. The amount of AIgG conjugated on LC microdroplets was determined using a calibration curve $\left(\lambda_{\max }=280 \mathrm{~nm}\right)$, which was drawn by taking standard solution of different concentrations of AIgG in PBS $(\mathrm{pH}=7.4)$ solution as required for analysis by Bradford method. The efficiency of conjugation of AIgG on cover glass immobilized LC microdroplets is found to be approximately $88 \%$, which resulted in conjugation of 2.95 ng (19.7 fmol) of AIgG per LC microdroplets or $8.8 \mu \mathrm{g}$ (58.7 pmol) of AIgG per glass cover (Table 2). The Bradford analysis of AIgG has clearly indicated that each LC microdroplets out of total immobilized microdroplets $(2988 \pm 2(n=3))$ on a cover glass was found to be conjugated with $2.95 \mathrm{ng}$ (19.7 fmol) of AIgG and $88 \%$ of AIgG was used in conjugation with slide cover glass immobilized LC microdroplets. The conjugation of AIgG

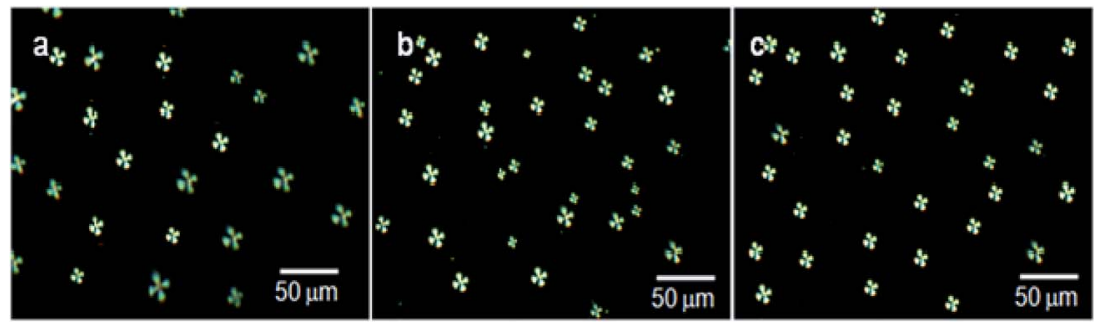

Fig. 4 Polarized light images of (a) free LC microdroplets, (b) slide cover glass immobilized LC microdroplets, and (c) slide cover glass immobilized AlgG-conjugated LC microdroplets. 
on cover glass immobilized LC microdroplets did not influence the orientation state of 5CB molecules but LC microdroplets remained in radial configuration as similar to pristine free LC microdroplets (Scheme 1a and Fig. 4a and c).

In order to confirm the conjugation of AIgG on cover glass immobilized LC microdroplets, the fluorescent AIgG-FITC conjugate was synthesized and used to conjugate on cover glass LC microdroplets. The formation of AIgG-FITC conjugate for immobilization on slide cover glass LC microdroplets was confirmed by comparing the UV-vis spectra of AIgG, FITC, and AIgG-FITC conjugate (Fig. 5). The UV spectra of FITC and AIgGFITC conjugate have shown an absorption bands at $495 \mathrm{~nm}$ (Fig. 5a) and $498 \mathrm{~nm}$ (Fig. 5c), respectively whereas, UV spectrum of pure AIgG did not show any absorption band around $498 \mathrm{~nm}$ (Fig. 5b).

After confirming the formation of AIgG-FITC conjugate by UV analysis, $50 \mu \mathrm{g}(\sim 34 \mathrm{pmol})$ of AIgG-FITC conjugate was added to a $100 \mathrm{~mL}$ round bottom flask containing slide-coverglass-immobilized LC microdroplets in $10 \mathrm{~mL}$ PBS solution and after that a calculated amount of EDC and NHS $(200 \mathrm{mg}$ each) was added along with $10 \mathrm{~mL}$ PBS solution. The resultant mixture of AIgG-FITC conjugate and slide-cover-glassimmobilized LC microdroplets was kept for about $4 \mathrm{~h}$ at $25{ }^{\circ} \mathrm{C}$. Finally, slide cover glass immobilized AIgG-FITC-

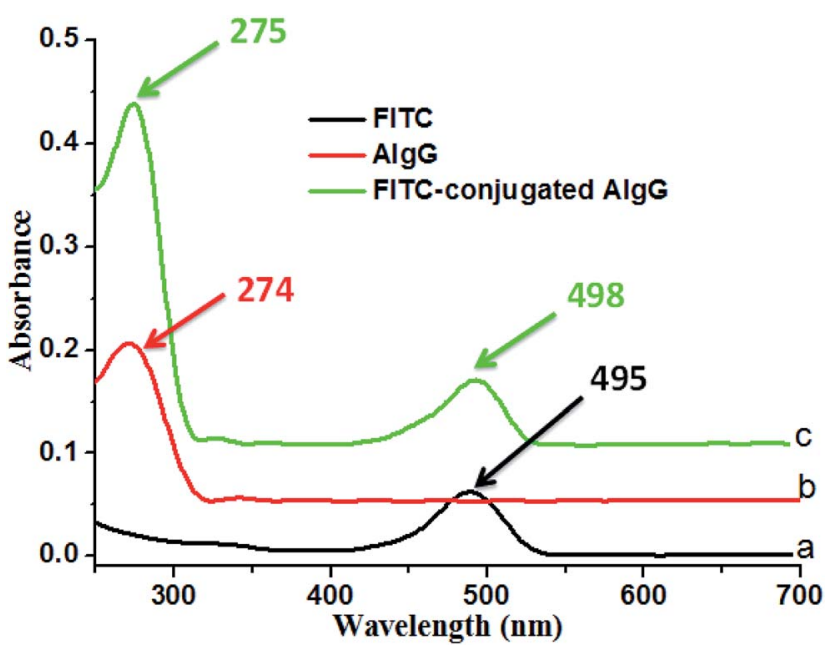

Fig. 5 UV-vis spectra of (a) FITC, (b) AlgG, and (c) AlgG-FITC conjugate. conjugated LC microdroplets were dialyzed with $\mathrm{PBS}(\mathrm{pH}=$ 7.4) solution using regenerated cellulose acetate membrane (MWCO, $12 \mathrm{kDa}$ ) and separated cover glass were washed with distilled water. The slide cover glass immobilized AIgG-FITCconjugated LC microdroplets were examined for orientational state of 5CB molecules by recording polarized light micrographs. The fluorescence imaging was carried out to confirm the anchoring of AIgG-FITC-conjugate on LC microdroplets. The anchoring of AIgG-FITC conjugate on LC microdroplets was $\sim 98 \%$ and did not influence the orientational state of $5 \mathrm{CB}$ molecules in LC microdroplets (Fig. 6b).

The 5CB molecules in LC microdroplets remained in radial configuration as found on immobilization of AIgG on LC microdroplets (Fig. 6a, Scheme S1 $\dagger$ ). The appearance of green fluorescence images of AIgG-FITC-conjugated LC microdroplets $\left(\lambda_{\mathrm{EX}}=519 \mathrm{~nm}\right)$ on slide cover glass has confirmed the conjugation of AIgG on LC microdroplets (Fig. 6c). The anchoring efficiency of AIgG-FITC conjugate on slide cover glass immobilized LC microdroplets is found to be $85 \%$ as similar to anchoring efficiency ( $88 \%$ ) of pure AIgG on slide cover glass immobilized LC microdroplets. The slight variation in anchoring efficiency of AIgG-FITC conjugate could be due to the decrease in anchoring sites of AIgG on its conjugation with FITC. The anchoring amount of AIgG-FITC conjugate on slide cover glass immobilized LC microdroplets was determined by applying Bradford method of analysis and using a calibration curve drawn by taking diluted solutions of AIgG-FITC conjugate with different concentration of AIgG. The fluorescence image of slide cover glass immobilized AIgG-FITC-conjugated LC microdroplets (Fig. 6c) has also indicated that none of AIgG molecule was present on unused amino groups on slide cover glass otherwise it would have been impossible to get clear green fluorescent images of AIgG-FITC-conjugated LC microdroplets as shown in Fig. 6c.

\section{Detection of IgG using slide cover glass immobilized AIgG- conjugated LC microdroplets}

After characterization of slide cover glasses for the immobilization of LC microdroplets and the loading efficiency of AIgG on slide cover glass immobilized LC microdroplets, the slide cover glass immobilized LC microdroplets were also evaluated to their sensitivity in detection of IgG antigen in PBS solution. The density of LC microdroplets immobilized on slide cover glass
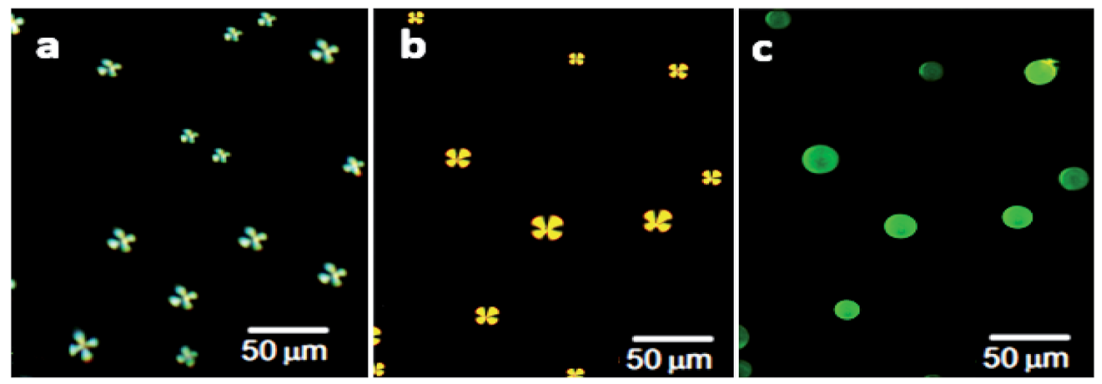

Fig. 6 Polarized light images of slide cover glass immobilized LC microdroplets conjugated with (a) AlgG-, and (b) AlgG-FITC-conjugate. (c) Fluorescence image of slide cover glass immobilized LC microdroplets conjugated with AlgG-FITC-conjugate. 
was found to be $3735 \pm 2.0(n=3)$ microdroplets per $\mathrm{cm}^{2}$ of slide cover glass $(2988 \pm 2.0(n=3)$ LC microdroplets per cover glass) and conjugated amount of AIgG on slide cover glass immobilized LC microdroplets was found to be $2.95 \mathrm{ng}$ (19.7 fmol) of AIgG per LC microdroplets or $8.8 \mu \mathrm{g}$ (58.7 pmol) of AIgG per slide cover glass. A molecule of AIgG has approximately covered $0.023 \mu \mathrm{m}^{2}$ area of LC microdroplets $(0.3 \%$ area of LC microdroplet). Each LC microdroplet immobilized on slide cover glass was saturated with $\sim 3.3 \times 10^{3} \mathrm{fmol}$ of IgG. The $5 \mathrm{CB}$ molecules in slide cover glass immobilized AIgG conjugated LC microdroplets were found to be in radial orientation (Fig. 7a, Scheme 1d) and changed to bipolar orientation (Fig. 7b, Scheme 1e) by antibody-antigen interaction forces, which were produced at the interface of LC microdroplets. The observed interfacial force of interactions was found to be quite sufficient and effective in causing orientational transitions in 5CB molecules in LC microdroplets.

These signals of optical transition from radial to bipolar in slide cover glass immobilized LC microdroplets were used to optimize the contact time of LC microdroplets for the detection

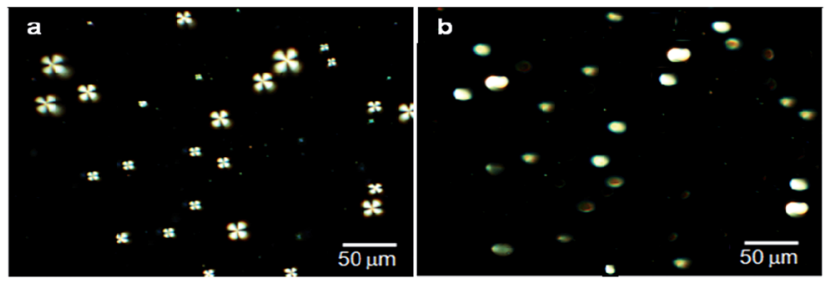

Fig. 7 Polarized light images of slide cover glass immobilized AlgG conjugated LC microdroplets showing (a) radial to (b) bipolar transition on interactions with $\mathrm{lg}$ G solution $(50 \mathrm{ng})$ at a contact time of $30 \mathrm{~min}$. of IgG antigen and for lower limit of detection (LOD) for IgG by slide cover glass immobilized LC microdroplets at different concentrations of IgG in PBS solution $(\mathrm{pH}=7.4)$ and in $10 \mathrm{wt} \%$ solution of FBS and blood plasma.

To optimize the contact time of LC microdroplets for the detection of IgG, the optical response of slide cover glass immobilized AIgG conjugated LC microdroplets was determined by keeping slide cover glass immobilized AIgG conjugated LC microdroplets in $10 \mathrm{~mL}$ PBS solution containing $50 \mathrm{ng}$ of IgG. The orientation state of 5CB molecules in LC microdroplets was examined at different contact times by recording their polarized light micrographs at three to four different positions on cover glass and average percentage of LC microdroplets showing orientation transition was determined.

The polarized light images of LC microdroplets recorded at a contact time of 5 min (Fig. 8b) have indicated that approximately $21 \%$ LC microdroplets $(627 \pm 0.5(n=3)$ microdroplets per cover glass) have shown radial to bipolar transitions and at a contact time of $15 \mathrm{~min}$ approximately $46 \%$ of LC microdroplets (1374 $\pm 0.8(n=3)$ microdroplets per cover glass) were transformed to bipolar orientations (Fig. 8c). After $45 \mathrm{~min}$ of contact time, all the cover glass immobilized LC microdroplets (2988 \pm 2 ( $n=3)$ microdroplets per cover glass) were found to be in bipolar orientation (Fig. 8d). This has indicated that $50 \mathrm{ng}$ of IgG was sufficient to transform all the cover glass immobilized LC microdroplets from radial to bipolar orientation within a contact time of 45 minutes. These contact time variation experiments were able to demonstrate that cover glass immobilized AIgG conjugated LC microdroplets were able to show detectable optical transition within a minimum contact time of $5 \mathrm{~min}$ in PBS solution containing $50 \mathrm{ng}$ of IgG. The fast response

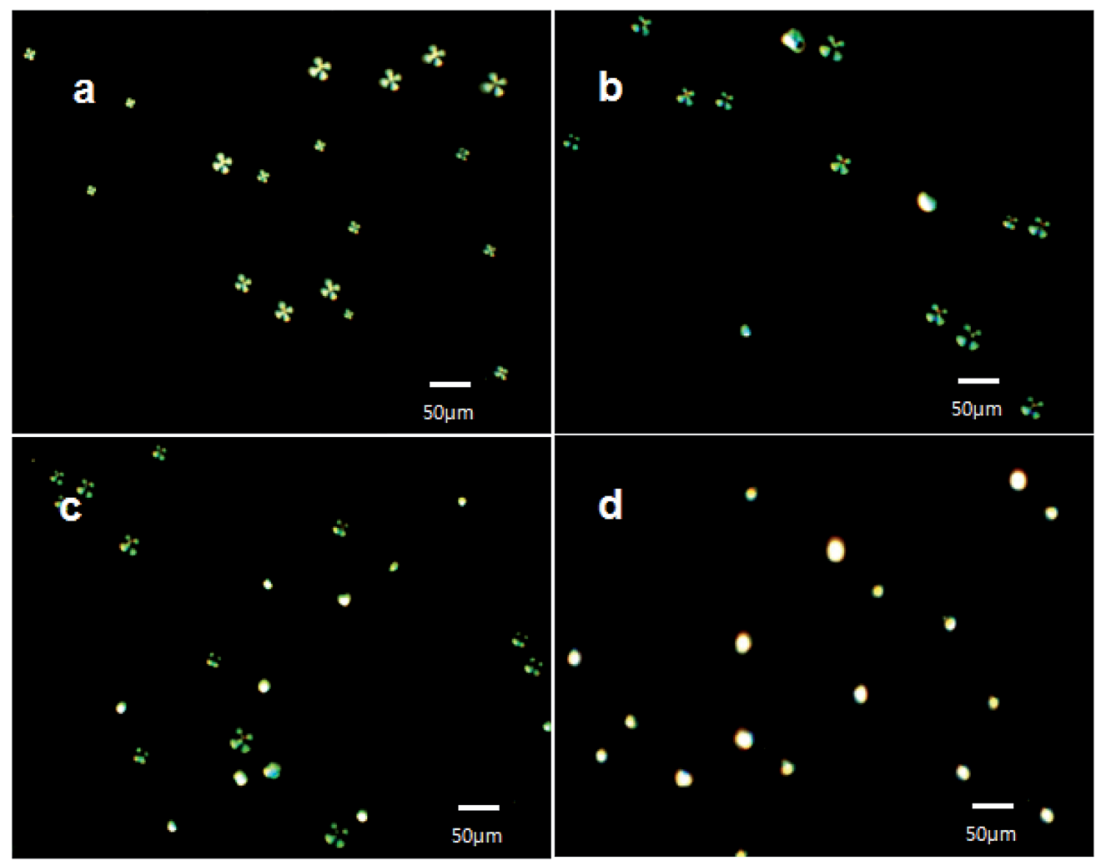

Fig. 8 Polarized light images of slide cover glass immobilized AlgG conjugated LC microdroplets showing radial to bipolar transition on

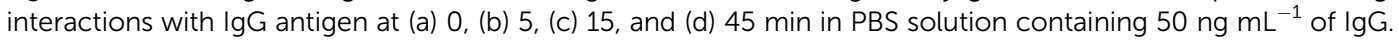


of $5 \mathrm{~min}$ for orientation transition in LC microdroplets is attributed to the positional stability of LC microdroplets on slide cover glass that provided opportunities to IgG antigen to interact effectively with LC microdroplets and for transduction of interfacial interaction forces to 5CB molecules in AIgGconjugated LC microdroplets to show orientation transition.

These results have also indicated that approximately $1.68 \pm$ $0.2 \mathrm{pg}$ of IgG was sufficient to cause orientation transition from radial to bipolar in a LC microdroplet immobilized on slide cover glass, which was loaded with $2.95 \pm 0.2 \mathrm{ng}$ of AIgG (Table 2 ). After evaluating the response time of cover glass immobilized LC microdroplets, the orientation response of cover glass immobilized LC microdroplets was also recorded on varying the amount of IgG antigen from 10-50 ng in PBS solution at a fixed contact time of $30 \mathrm{~min}$ (Table 2). The polarized light micrographs of cover glass immobilized LC microdroplets have shown all the LC microdroplets with radial configuration, on keeping for 30 minutes in a solution containing $10 \mathrm{ng} \mathrm{mL} \mathrm{mL}^{-1}$ of IgG antigen (Fig. 9a). This result has indicated that the IgG present in PBS solution was sufficiently low to show detectable orientation transition in LC microdroplets even after contacting IgG antigen for $30 \mathrm{~min}$ in PBS solution. The orientation response of 5CB molecules in cover glass immobilized LC microdroplets after contacting for $30 \mathrm{~min}$ in a PBS solution containing $25 \mathrm{ng} \mathrm{mL} \mathrm{mL}^{-1}$ of IgG was also recorded from which it was observed that $25 \mathrm{ng} \mathrm{mL} \mathrm{mL}^{-1}$ amount of IgG in PBS solution was able to cause radial to bipolar transition in 57\% slide cover glass immobilized LC microdroplets (1704 $\pm 0.4(n=3)$ microdroplets per cover glass) (Fig. 9b). Thus interaction force, which was produced in presence of $25 \mathrm{ng} \mathrm{mL}{ }^{-1}$ concentration of IgG in PBS solution was quite sufficient to create detectable orientation transition in cover glass immobilized LC microdroplets within a contact time of $30 \mathrm{~min}$ (Fig. 9b) in comparison to interactions force, which were produced in presence of $10 \mathrm{ng}$ $\mathrm{mL}^{-1}$ concentration of IgG antigen (Fig. 9a). To determine the minimum amount of IgG that could induce $100 \%$ transition in cover glass immobilized LC microdroplets, the optical response of LC microdroplets was also recorded by keeping cover glass immobilized LC microdroplets for 30 minutes in PBS solution containing $50 \mathrm{ng} \mathrm{mL} \mathrm{m}^{-1}$ concentration of IgG. The polarized light micrographs of these LC microdroplets have shown 100\% transition from radial to bipolar $(2988 \pm 2(n=3)$ microdroplets per cover glass) (Fig. 9c). This has indicated that PBS solution containing $50 \mathrm{ng} \mathrm{mL} \mathrm{mL}^{-1}$ concentration of IgG was able to create sufficient interfacial forces that could induce $100 \%$ radial to bipolar transitions in cover glass immobilized LC microdroplets having a density of 3735 LC microdroplets per $\mathrm{cm}^{2}$. These results have clearly indicated that the cover glass immobilized LC microdroplets could reveal detectable orientation transition, if a minimum amount of $1.68 \pm 0.2 \mathrm{pg}$ per microdroplet of IgG antigen is present in PBS solution and each LC microdroplets is conjugated with $2.95 \pm 0.2 \mathrm{ng}$ of AIgG but these LC microdroplets failed to show optical transition, if the amount of IgG antigen in test solution was below the lower limit of detection for IgG antigen $(<1.68 \pm 0.2 \mathrm{pg}$ per microdroplet) in PBS solution. Since in a PBS solution containing $10 \mathrm{ng} \mathrm{mL} \mathrm{mL}^{-1}$ of IgG, the amount of IgG per LC microdroplet was low $(0.67 \pm 0.02 \mathrm{pg}$ per LC microdroplet) than the lower limit of detection of LC microdroplets $(<1.68 \pm 0.2 \mathrm{pg}$ per microdroplet), hence the LC microdroplets were unable to show optical transition (Fig. 9a) even after a contact time of 30 minutes. The detection of IgG antigen within a concentration range of $10-50 \mathrm{ng} \mathrm{mL} \mathrm{m}^{-1}(0.67-$ $2.78 \pm 0.2 \mathrm{pg}$ per microdroplet) is found useful in determining the lower limit of detection of IgG by slide cover glass immobilized LC microdroplets in PBS solution (Table 2). The lower detection limit of IgG by cover glass immobilized LC microdroplets is found to be quite low $(<1.68 \pm 0.2 \mathrm{pg}$ per microdroplet or $25 \mathrm{ng} \mathrm{mL}^{-1}$ ) (Table 2 ) in comparison to unbound free LC microdroplets or in comparison other methods used for the detection of antigens in a solution, ${ }^{\mathbf{3 8 , 4 7 , 4 8}}$ where lower detection by LC microdroplets is reported to be $5 \mu \mathrm{g} \mathrm{mL}{ }^{-1} .^{48}$

\section{Detection of IgG in FBS and blood plasma using slide cover glass immobilized AIgG-conjugated LC microdroplets}

To evaluate the feasibility of slide cover glass immobilized AIgG conjugated LC microdroplets for detection of IgG in biological fluids, the cover glass-immobilized AIgG-conjugated LC microdroplets were used for the detection of IgG in a solution containing $10 \mathrm{wt} \%$ of FBS or blood plasma. As similar to PBS solution, the optical response of cover glass immobilized LC microdroplets was determined at different time intervals and taking different amount of IgG to determine the effect of medium on response time and on lower limit of detection of IgG by cover glass immobilized LC microdroplets. The response of cover glass immobilized LC microdroplets in $10 \mathrm{wt} \%$ solution of FBS was evaluated at 0, 30, 60, and 90 min (Fig. 10).

The response of cover glass immobilized AIgG conjugated LC microdroplets in solution containing $10 \mathrm{ng} \mathrm{mL}{ }^{-1}$ amount of IgG
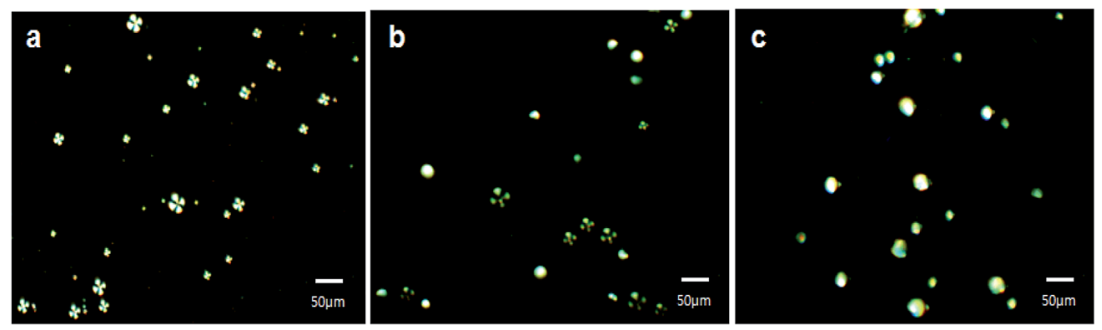

Fig. 9 Polarized light images of slide cover glass immobilized AlgG conjugated LC microdroplets showing radial to bipolar transition at a contact time of 30 min in PBS solution containing (a) 10, (b) 25, and (c) $50 \mathrm{ng} \mathrm{mL}^{-1}$ of IgG antigen. 

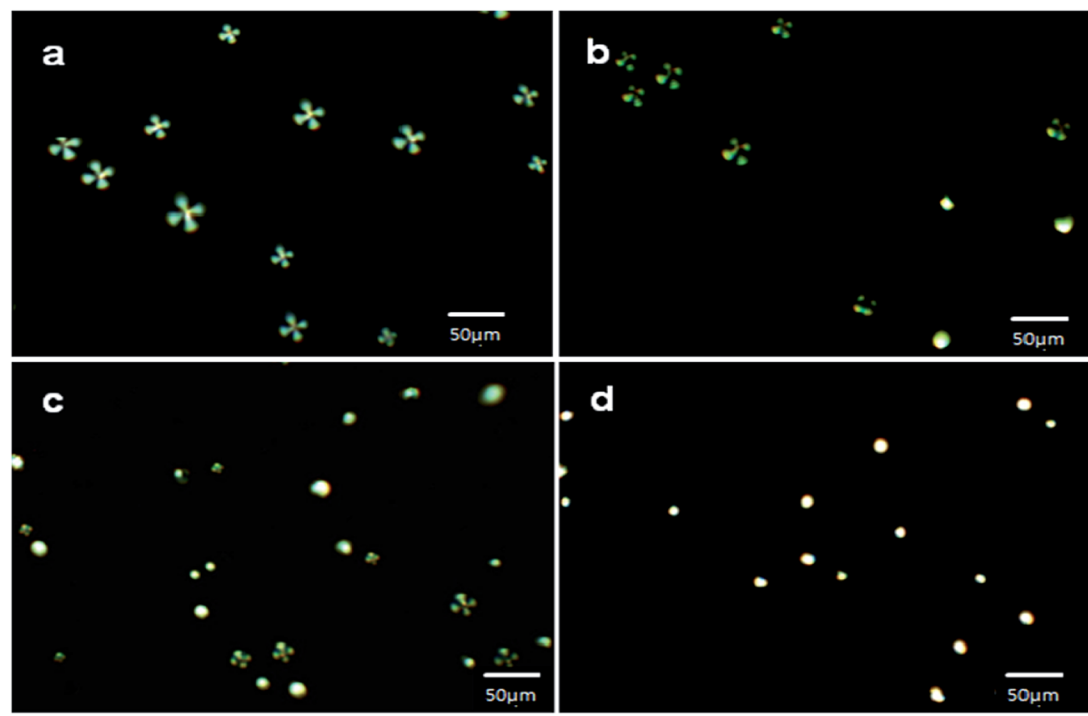

Fig. 10 Polarized light images of cover glass immobilized AlgG conjugated LC microdroplets showing radial to bipolar transition on interactions with IgG at (a) 0 , (b) 30, (c) 60, and (d) $90 \mathrm{~min}$ in 10 wt\% solution of FBS solution containing $50 \mathrm{ng} \mathrm{mL}^{-1}$ of IgG antigen.

and $10 \mathrm{wt} \%$ of FBS was found to be delayed infinitely. The delayed response of LC microdroplets to $60 \mathrm{~min}$ in solution containing $25 \mathrm{ng} \mathrm{mL} \mathrm{m}^{-1}$ amount of IgG and $10 \mathrm{wt} \%$ of FBS has been attributed to the presence of protein molecules in these media, which reduced the diffusion of IgG molecules to interact with LC microdroplets and to produce optical signal $(\geq 50 \%$ transition in LC microdroplets) before a contact time of 60 minutes. The orientational transition from radial to bipolar in AIgG conjugated LC microdroplets was found to be $30 \%$ (897 \pm 0.4 $(n=3)$ LC microdroplets per cover glass) (Fig. 10b), 69\% $(2062 \pm 1.3(n=3)$ LC microdroplets per cover glass) (Fig. 10c), and $100 \%(2988 \pm 2.1(n=3)$ LC microdroplets per cover glass) (Fig. 10d) at a contact time of $30 \mathrm{~min}, 60 \mathrm{~min}$ and $90 \mathrm{~min}$ respectively in solution containing $50 \mathrm{ng} \mathrm{mL}^{-1}$ of IgG and 10 $\mathrm{wt} \%$ amount of FBS. The time dependent response of slide cover glass immobilized AIgG conjugated LC microdroplets in solution containing $50 \mathrm{ng} \mathrm{mL}{ }^{-1}$ amount of IgG and $10 \mathrm{wt} \%$ amount of blood plasma was found to be almost same as found in solution with $10 \mathrm{wt} \%$ amount of FBS (Fig. 10). The slide cover glass immobilized AIgG conjugated LC microdroplets have shown $0 \%$ ( 0 microdroplets per cover glass), $28 \%$ (837 \pm 0.18 ( $n$ =3) microdroplets per cover glass), and 65\% (1943 $\pm 1.90(n=$ 3) microdroplets per cover glass) radial to bipolar transitions at a contact time of $0 \mathrm{~min}, 30 \mathrm{~min}$ and $60 \mathrm{~min}$ respectively in solution containing $50 \mathrm{ng} \mathrm{mL}^{-1}$ amount of IgG and $10 \mathrm{wt} \%$ amount of blood plasma. The slide cover glass immobilized AIgG conjugated LC microdroplets have shown 100\% (2988 \pm $1.9(n=3))$ radial to bipolar transition at a contact time of $90 \mathrm{~min}$ in solution containing $50 \mathrm{ng} \mathrm{mL} \mathrm{m}^{-1}$ amount of IgG and 10 wt $\%$ amount of blood plasma, which is almost same as found in solution with $10 \mathrm{wt} \%$ amount of FBS (Fig. 10d). A detectable response $(\sim 20 \%$ conversion in microdroplets) in slide cover glass immobilized LC microdroplets in solution containing 50 ng $\mathrm{mL}^{-1}$ amount of IgG and $10 \mathrm{wt} \%$ amount of FBS or blood plasma was found within a contact time of $5 \mathrm{~min}$ as found in PBS solution (Fig. 9c).
In addition to time dependent response of slide cover glass immobilized LC microdroplets, the response of LC microdroplets was also evaluated in solution containing different amount of IgG and $10 \mathrm{wt} \%$ amount of FBS or blood plasma. The concentration variation of IgG was carried out to evaluate the lower limit of detection of IgG in solution with $10 \mathrm{wt} \%$ amount of FBS or blood plasma as it was evaluated in PBS solution (Fig. 9). In comparison to PBS, a contact time of 60 min was chosen for studying the effect of IgG concentration variation in solution with $10 \mathrm{wt} \%$ amount of FBS or blood plasma to get detectable optical transition $(\geq 50 \%)$ in LC microdroplets. The concentration of IgG was varied from $10-50 \mathrm{ng} \mathrm{mL}^{-1}$ as similar to PBS solution (Fig. 9, Table 2). The slide cover glass immobilized LC microdroplets in solution containing $10 \mathrm{ng} \mathrm{mL}^{-1}$ amount of IgG and $10 \mathrm{wt} \%$ amount of FBS did not show any orientational transition even after a contact time of $90 \mathrm{~min}$ (Fig. 11a). Similarly, no optical transition was observed in LC microdroplets in solution containing $10 \mathrm{ng} \mathrm{mL} \mathrm{m}^{-1}$ amount of IgG and $10 \mathrm{wt} \%$ amount of blood plasma. Furthermore, approximately $52 \%(1554 \pm 2.1(n=3)$ microdroplets per cover glass $)$ LC microdroplets have shown radial to bipolar transition in solution containing $25 \mathrm{ng} \mathrm{mL} \mathrm{m}^{-1}$ amount of IgG and $10 \mathrm{wt} \%$ amount of FBS (Fig. 11b, Table 2). On further increasing the amount of IgG to $50 \mathrm{ng} \mathrm{mL} \mathrm{m}^{-1}$ in solution, which was containing $10 \mathrm{wt} \%$ amount of FBS, $100 \%(2988 \pm 2.2(n=3)$ microdroplets per cover glass) LC microdroplets were able to show orientation transition from radial to bipolar within a contact time of $90 \mathrm{~min}$ (Fig. 11c) as compared to LC microdroplets in PBS solution, which were contacting $50 \mathrm{ng} \mathrm{mL} \mathrm{m}^{-1}$ amount of IgG and were able to show radial to bipolar transition in $100 \%(2988 \pm 2.2(n=3)$ microdroplets per cover glass) LC microdroplets within a contact time of $30 \mathrm{~min}$ (Fig. 9c).

On comparing the concentration dependence response of cover glass immobilized LC microdroplets for detection of IgG in solution with $10 \mathrm{wt} \%$ amount of FBS (Fig. 11) with the response of LC microdroplets in solution of PBS (Fig. 9), it is 


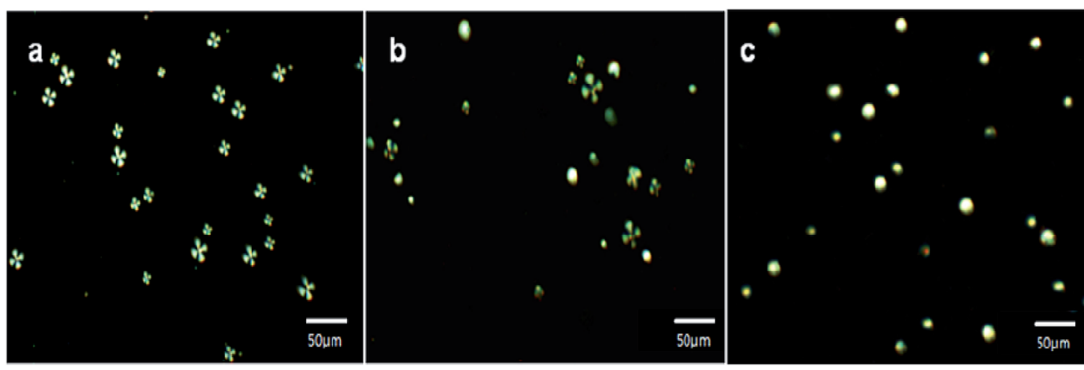

Fig. 11 Polarized light images of cover glass immobilized AlgG conjugated LC microdroplets showing radial to bipolar transition at a contact time of 90 min in 10 wt\% of FBS solution having $\operatorname{lgG}$ (a) 10 , (b) 25 , and (c) $50 \mathrm{ng} \mathrm{mL}^{-1}$ of IgG antigen.

clear that $25 \mathrm{ng} \mathrm{mL} \mathrm{m}^{-1}$ of IgG was sufficient $(1.68 \pm 0.2 \mathrm{pg}$ per microdroplet) to produce a detectable signal $(\geq 50 \%)$ in solution with $10 \mathrm{wt} \%$ amount of FBS solution as found with PBS solution (Table 2). Thus lower limit of detection for IgG in solution with $10 \mathrm{wt} \%$ amount of FBS was same as was found with PBS. The observed trends in optical signal in cover glass immobilized microdroplets on varying the amount of IgG from 10-50 ng $\mathrm{mL}^{-1}$ in solution with $10 \mathrm{wt} \%$ amount of FBS (Fig. 11) was found to be same as was found for the detection of IgG in PBS solution (Fig. 9) except a variation in contact time from $30 \mathrm{~min}$ to $60 \mathrm{~min}$ (Table 2).

This variation in contact time from 30 to 60 minutes was attributed to the presence of protein molecules in solution with $10 \mathrm{wt} \%$ amount of FBS in comparison to PBS solution in which the mobility of IgG molecules remained uninterrupted and they were able to interact and bind quickly with AIgG-conjugated LC microdroplets within a contact time of $30 \mathrm{~min}$.

The response of slide cover glass immobilized LC microdroplets on varying the amount of IgG from $10-50 \mathrm{ng} \mathrm{mL}^{-1}$ was also studied at a contact time of $60 \mathrm{~min}$ in solution with $10 \mathrm{wt} \%$ amount of blood plasma (Table 2). At low concentration of IgG $\left(10 \mathrm{ng} \mathrm{mL}^{-1}\right.$ ), none of the LC microdroplet was able to show orientational transition in solution $10 \mathrm{wt} \%$ amount of blood plasma. However, on further increasing the amount of IgG to 25 $\mathrm{ng} \mathrm{mL}{ }^{-1}$ in solution with $10 \mathrm{wt} \%$ amount of blood plasma, $48 \%$ $(1435 \pm 1.8(n=3)$ microdroplets per cover glass) LC microdroplets were able to shown radial to bipolar transitions (Table 2), which were comparable to the orientation transitions as observed in solution with $10 \mathrm{wt} \%$ amount of FBS (Fig. 11b) as well as with PBS solution (Fig. 9b). This has confirmed that concentration dependence sensitivity of slide cover glass immobilized LC microdroplets to IgG in solution with $10 \mathrm{wt} \%$ amount of blood plasma remained same as was found in solution with $10 \mathrm{wt} \%$ amount of FBS and PBS solution (Table 2). The slide cover glass immobilized LC microdroplets in solution containing $50 \mathrm{ng} \mathrm{mL} \mathrm{mL}^{-1}$ amount of IgG and $10 \mathrm{wt} \%$ amount of blood plasma were able to show orientational transition in $100 \%(2988 \pm 2.2(n=3)$ microdroplets per cover glass) LC microdroplets as similar to PBS solution (Fig. 9c) or solution with $10 \mathrm{wt} \%$ amount of FBS (Fig. 11c). These results have clearly indicated that the sensitivity of cover glass immobilized LC microdroplets in solution with $10 \mathrm{wt} \%$ amount of FBS or blood plasma remained almost same as was found in PBS solution. Therefore, cover glass immobilized LC microdroplets may be used for the detection of IgG antigen in biological fluids having IgG concentration slightly greater than the lower limit of detection (1.68 $\mathrm{pg} \mathrm{mL}^{-1}$ ) of IgG by slide cover glass immobilized AIgG conjugated LC microdroplets. Though slide cover glass immobilization has shown better sensitivity in detection of IgG antigen but there must be a control on size distribution of LC microdroplets so that cover glass with high density of LC microdroplets are obtained. The sensitivity of LC microdroplet decreased significantly on taking LC microdroplets of smaller size $(<15 \mu \mathrm{m})$ or more than $30 \mu \mathrm{m}$ in diameter. The sensitivity of the cove glass was also deviated on taking high concentration of IgG (>50 ng mL $\mathrm{mL}^{-1}$ ) or taking IgG below the lower limit of detection for IgG in liquid media.

\section{Conclusions}

The slide cover glass immobilized AIgG conjugated LC microdroplets have been used successfully for the detection of rabbit antigen (IgG) in PBS solution and other media such as solution with $10 \mathrm{wt} \%$ amount of FBS or blood plasma. The immobilization of LC microdroplets on slide cover glasses was carried out successfully using amino functionalized slide cover glass to provide stability and orientation to LC microdroplets. This technique of LC microdroplets immobilization on slide cover glass has increased the lower limit of detection of LC microdroplets for IgG down to $1.68 \pm 0.2 \mathrm{pg}$ per microdroplet $(25 \mathrm{ng}$ $\mathrm{mL}^{-1}$ ), which might be a significant improvement in controlling the efficiency of LC microdroplets in detection of IgG in PBS solution in comparison to liquid crystal detection of antigen or proteins on planar solid surfaces. ${ }^{48}$ The cover glass immobilized LC microdroplets were able to provide better interactions of AIgG with IgG in liquid media to produce detectable optical signal in LC microdroplets ( $\geq 20 \%$ ) at low concentration of IgG. The slide cover glass immobilized LC microdroplets were found suitable for detection of IgG in solution with $10 \mathrm{wt} \%$ amount of FBS or blood plasma within a concentration rang of 10-50 ng $\mathrm{mL}^{-1}$ of IgG and with almost same lower limit of detection (1.68 $\pm 0.2 \mathrm{pg}$ per microdroplet or $25 \mathrm{ng} \mathrm{mL}^{-1}$ ) for IgG except for a delayed response time of $60 \mathrm{~min}$ due to frictional interferences from the protein molecules present in FBS or blood plasma. These results have given an idea for controlling the optical sensitivity of 5CB molecules by the technique of immobilization on solid cover glasses in comparison to bulk immobilization of 5CB molecules on solid surfaces as layered 
structures. The 5CB molecules in the form of microdroplets are able to provide better opportunities to interact with antigens or other foreign molecules and were able to produce detectable optical signals by textural variation in liquid crystal molecules without using other methods of orientation transitions. The immobilization of LC microdroplets on slide cover glass has helped in improving the interfacial forces, which are presumed to be responsible to cause orientation transition in 5CB molecules aligned at the curved interfacial surface of LC microdroplets. The results reported in this study are potentially useful to develop biosensors for naked eye detection of disease-related pathogens and for detection of circulating bio-markers in biological fluids.

\section{Author contributions}

Ms Huan Yue, was a student of Master of Engineering at the Department of Polymer Science and Engineering, Kyungpook National University, Daegu, South Korea, and made substantial contributions in designing the experiment and acquisition of data. Ms So Jung Park is a student of Master of Engineering at the Department of Polymer Science and Engineering, Kyungpook National University, Daegu, South Korea, and helped in revision of this manuscript. Prof. Kailash Chandra Gupta participated in critical discussions on the contents and drafting of the manuscript for publication. Prof. Soo-Young Park and Inn-Kyu Kang gave the final approval after evaluating and discussing the contents.

\section{Conflict of interest}

The authors declare no competing financial interest.

\section{Acknowledgements}

This work was supported by the Basic Science Research Program through the National Research Foundation (NRF) funded by the Ministry of Education (No. 2016R1D1A1A09918166 and No. 2011-0020264). One of the authors, Prof. K. C. Gupta, would like to thank Prof. Inn-Kyu Kang for sponsoring his visit to Department of Polymer Science and Engineering at Kyungpook National University as visiting professor for collaborative research work.

\section{References}

1 Y. Zhang, Y. Guo, Y. Xianyu, W. Chen, Y. Zhao and X. Jiang, Adv. Mater., 2013, 25, 3802-3819.

2 E. C. Dreaden and M. A. El-Sayed, Acc. Chem. Res., 2012, 45, 1854-1865.

3 B. Dou, J. Yang, K. Shi, R. Yuan and Y. Xiang, Biosens. Bioelectron., 2016, 83, 156-161.

4 L. T. Jia, Y. C. Zhang, J. Li, Y. Tian and J. F. Li, Clin. Transl. Oncol., 2016, 18, 233-239.

5 H. Zhang, Q. Zhao, X. F. Li and X. C. Le, Analyst, 2007, 132, 724-737.
6 K. Shin, J. H. Cho, M. Y. Yoon and H. Chung, Anal. Chem., 2016, 88, 3465-3470.

7 N. Lu, A. Gao, P. Dai, H. Mao, X. Zuo, C. Fan, Y. Wang and T. Li, Anal. Chem., 2015, 87, 11203-11208.

8 M. N. Ma, Y. Zhuo, R. Yuan and Y. Q. Chai, Anal. Chem., 2015, 87, 11389-11397.

9 L. Switzar, M. Giera and W. M. A. Niessen, J. Proteome Res., 2013, 12, 1067-1077.

10 J. M. Spraggins, D. G. Rizzo, J. L. Moore, K. L. Rose, N. D. Hammer, E. P. Skaar and R. M. Caprioli, J. Am. Soc. Mass Spectrom., 2015, 26, 974-985.

11 S. Imrich, M. Hachmeister and O. Gires, Cell Adhes. Migr., 2012, 6, 30-38.

12 R. Du, L. Zhu, J. Gan, Y. Wang, L. Qiao and B. Liu, Anal. Chem., 2016, 88, 6767-6772.

13 J. R. Lee, J. Lee, S. K. Kim, K. P. Kim, H. S. Park and W. S. Yeo, Angew. Chem., Int. Ed., 2008, 47, 9518-9521.

14 H. Hatanaka, T. Yasukawa and F. Mizutani, Anal. Chem., 2011, 83, 7207-7212.

15 T. G. Fernandes, S. J. Kwon, M. Y. Lee, D. S. Clark, J. M. S. Cabral and J. S. Dordick, Anal. Chem., 2008, 80, 6633-6639.

16 L. Dexlin, J. Ingvarsson, B. Frendeus, C. A. K. Borrebaeck and C. Wingren, J. Proteome Res., 2008, 7, 319-327.

17 G. Stybayeva, O. Mudanyali, S. Seo, J. Silangcruz, M. Macal, E. Ramanculov, S. Dandekar, A. Erlinger, A. Ozcan and A. Revzin, Anal. Chem., 2010, 82, 3736-3744.

18 K. Kato, T. Ishimuro, Y. Arima, I. Hirata and H. Iwata, Anal. Chem., 2007, 79, 8616-8623.

19 K. Lee, K. C. Gupta, S. Y. Park and I. K. Kang, J. Mater. Chem. $B, 2016,4,704-715$.

20 S. H. Yoon, K. C. Gupta, J. S. Borah, S. Y. Park, Y. K. Kim, J. H. Lee and I. K. Kang, Langmuir, 2014, 30, 10668-10677.

21 T. Kreising, R. Hoffmann and T. Zuchner, Anal. Chem., 2011, 83, 4281-4287.

22 F. Zuo, Z. Liao, C. Zhao, Z. Qin, X. Li, C. Zhang and D. Liu, Chem. Commun., 2014, 50, 1857-1860.

23 M. L. Bungabong, P. B. Ong and K. L. Yang, Sens. Actuators, $B, 2010,148,420-426$.

24 D. Hartono, S. L. Lai, K. L. L. Yang and L. Y. L. Yung, Biosens. Bioelectron., 2009, 24, 2289-2293.

25 H. J. VanTreeck, D. R. Most, B. A. Grinwald, K. A. Kupcho, A. Sen, M. D. Bond and B. R. Acharaya, Sens. Actuators, B, 2011, 158, 104-110.

26 P. Baptista, E. Pereira, P. Eaton, D. Doria, A. A. Miranda, I. Gomes, P. Quaresma and R. Franco, Anal. Bioanal. Chem., 2008, 391, 943-950.

27 X. Cui, M. Liu and B. Li, Analyst, 2012, 137, 3293-3299.

28 J. M. Nam, C. S. Thaxton and C. A. Mirkin, Science, 2003, 301, 1884-1886.

29 S. Pathak, S. K. Choi, N. Arnheim and M. E. Thompson, J. Am. Chem. Soc., 2001, 123, 4103-4104.

30 X. Wu, H. Liu, J. Liu, K. N. Haley, J. A. Treadway, J. P. Larson, N. Ge, F. Peale and M. P. Bruchez, Nat. Biotechnol., 2002, 21, 41-46.

31 X. Gao, Y. Cui, R. M. Levenson, L. W. K. Chung and S. Nie, Nat. Biotechnol., 2004, 22, 969-976. 
32 T. A. Taton, C. A. Mirkin and R. L. Letsinger, Science, 2000, 289, 1757-1760.

33 L. Zhu, S. Ang and W. T. Liu, Appl. Environ. Microbiol., 2004, 70, 597-598.

34 S. Dwarakanath, J. G. Bruno, A. Shastry, T. Phillips, A. A. John, A. John, A. Kumar and L. D. Stephenson, Biochem. Biophys. Res. Commun., 2004, 325, 739-743.

35 H. Xu, X. Bi, X. Ngo and K. L. Yang, Analyst, 2009, 134, 911915.

36 S. Sivakumar, K. L. Yang, J. K. Gupta, N. L. Abbott and F. Caruso, Adv. Funct. Mater., 2009, 19, 2260-2265.

37 C. Y. Xue and K. L. Yang, Langmuir, 2008, 24, 563-567.

38 C. S. Xue, S. A. Khan and K. L. Yang, Adv. Funct. Mater., 2008, 21, 198-202.

39 N. A. Lockwood, J. K. Gupta and N. L. Abbott, Surf. Sci. Rep., 2008, 63, 255-293.

40 O. Tongcher, R. Siegel and K. Landfester, Langmuir, 2006, 22, 4504-4511.
41 K. A. Simon, P. Sejwal, R. B. Gerecht and Y. Y. Luk, Langmuir, 2007, 23, 1453-1458.

42 E. Tjipto, K. D. Cadwell, J. F. Quinn, A. P. Johnson, N. L. Abbott and F. Caruso, Nano Lett., 2006, 2243-2248.

43 J. K. Gupta, S. Sivakumar, F. Caruso and N. L. Abbott, Angew. Chem., Int. Ed., 2009, 121, 1680-1683.

44 S. Sivakumar, J. K. Gupta, N. L. Abbott and F. Caruso, Chem. Mater., 2008, 20, 2063-2065.

45 A. Munir, J. Wang and H. S. Zhou, IET Nanobiotechnol., 2009, 3, 55-64.

46 K. S. Kim and J. K. Park, Lab Chip, 2005, 5, 657-664.

47 R. Birchall, S. Ulijn and A. Webb, Chem. Commun., 2008, 2861-2863.

48 C. Y. Xue and K. L. Park, Langmuir, 2008, 24, 563-567.

49 M. B. Haddada, J. Blanchard and S. Casale, Gold Bull., 2013, 46, 335-341.

50 S. M. Kanan, W. T. Y. Tze and C. P. Tripp, Langmuir, 2002, 18, 6623-6627. 